\title{
A comparison between the effects of morphine on the rewarding and aversive properties of lateral hypothalamic and central gray stimulation
}

\author{
SUSAN SCHENK, TERRENCE WILLIAMS, ALAIN COUPAL, and PETER SHIZGAL \\ Center for Research on Drug Dependence, Concordia University, Montreal, Quebec H3G 1J9, Canada
}

\begin{abstract}
Rats were trained to initiate and terminate lateral hypothalamic or dorsal raphe stimulation. The effects of various doses of morphine on self-stimulation (SS) and stimulation escape (SE) were assessed for $7 \mathrm{~h} /$ day. The drug produced a strong attenuation of $\mathrm{SE}$ at both placements. This effect did not show tolerance with repeated injections of morphine. Similarly, SS was depressed for 1-2 $\mathrm{h}$ following drug injection. This effect did tolerate with chronic treatment. A drug-produced facilitation of SS was observed in some subjects, although the magnitude of this effect varied across animals. The data are interpreted to suggest that morphine acts on both the substrate for SS and the substrate for SE, but in opposite directions.
\end{abstract}

The well-documented effects of morphine on lateral hypothalamic (LH) self-stimulation (SS) have been recently reviewed by Esposito and Kornetsky (1978). The drug typically produces a biphasic effect; an initial depression lasts for $2-3 \mathrm{~h}$ postinjection and is followed by a facilitatory effect. The depression tolerates after several days of injections, while the facilitation occurs sooner in time and grows in magnitude with chronic treatment.

Some investigators have studied morphine's modulation of the rewarding properties of brain stimulation at sites other than the LH. While facilitation following various drug doses has been observed at medial frontal cortex (Lorens, 1976) and dorsal brainstem (Jackler, Steiner, Bodnar, Ackerman, Nelson, \& Ellman, 1979) placements, the time course and duration of these effects are somewhat different from those reported in the $\mathrm{LH}$, implying some anatomical specificity for the drug's positively reinforcing properties. Also, placement-specific facilitatory effects have been reported in the dorsal raphe (DR) (Liebeman \& Segal, 1977).

The effects of morphine on escape from aversive stimulation have been less intensively studied than have the drug's effects on SS. Marcus and Kornetsky (1974) reported morphine-produced increases in intensity thresholds for escape from reticular formation stimulation. Pert (1975) replicated these findings and found tolerance to these depressive effects, suggesting

This work was supported by F.C.A.C. Grant EQ-09 from the Ministère de l'Education du Québec. The authors' complete address is: Center for Research on Drug Dependence, Department of Psychology, Concordia University, 1455 de Maisonneuve Blvd. West, Montreal, Quebec H3G 1J9, Canada. that the modulation by morphine of the aversive effects of brain stimulation was related to the drug's analgesic properties. It has further been suggested that this analgesic action might block the aversive consequences that accompany SS, thus producing a net increase in the rewarding properties of the stimulation (Baltzer, Levitt, \& Furby, 1977).

The present experiments were designed to further investigate and compare the chronological evolution of morphine's effects on the rewarding and aversive properties of LH and DR stimulation. The DR has been found to support SS (Simon, Le Moal, \& Cardo, 1976) and SE (Steiner, Bodnar, Ackerman, \& Ellman, 1973). There is little transfer of learning between sites in the LH and the DR (Steiner et al., 1973). This would suggest that there is a difference in the states evoked by stimulation of each site and in their neural substrates. There is also pharmacological evidence that serotonergic blockade differentially affects LH (Robertson, Kucharczyk, \& Mogenson, 1977) and DR (Simon et al., 1976) SS, although conflicting findings have been reported (Poschel \& Ninteman, 1971).

There is also a large body of evidence implicating the DR in morphine analgesia (Mayer \& Price, 1976). If morphine-produced attenuation of escape responding is related to analgesia, the drug effect should be pronounced at the DR placement.

Response-rate measures of SS performance have been shown to be subject to basement and ceiling effects (Yeomans, 1975). Therefore, we chose, instead, a frequency threshold technique that has been reported to circumvent these problems (Yeomans, 1975). We have also found that the frequency threshold scaling works well for stimulation-escape (SE) (Bielajew \& 
Shizgal, in press). Frequency, rather than intensity, thresholds were used in order to hold constant the size of the effective stimulation field and, hence, the population of directly stimulated neurons.

The concurrent observation of drug-produced changes in SS and SE may provide an indication of nonspecific performance artifacts. Such artifacts may be suspected when drug-induced changes in behavior are in the same direction.

\section{EXPERIMENT 1}

The first experiment was designed to chart the time course of morphine's effects on the aversive consequences of $\mathrm{LH}$ stimulation. These effects were then compared with drug-produced changes in SS.

\section{Method}

\section{Subjects}

Male Sprague-Dawley rats (Canadian Breeding Farms, St. Constant, Quebec), weighing approximately $350 \mathrm{~g}$ at the time of surgery, were housed individually and maintained on ad-lib food and water throughout the experiment. Under sodium pentobarbital anesthesia $(60 \mathrm{mg} / \mathrm{kg}$, ip), monopolar stimulation electrodes were stereotaxically aimed at the lateral hypothalamus (incisor bar at $+5 \mathrm{~mm}$ relative to the interaural line; $A P,-.4 \mathrm{~mm}$; lat, $-1.7 \mathrm{~mm} ; 8.0 \mathrm{~mm}$ below the dura). Electrodes were 00 stainless steel insect pins insulated with Formvar to within $.5 \mathrm{~mm}$ of the tip. Six stainless steel jeweler's screws served as the current return. The electrode shaft was fixed to the skull with dental acrylic.

\section{Training}

Self-stimulation. After at least 5 days' recovery from surgery, the rats were trained by successive approximation to self-stimulate in a wooden box $(25 \times 28 \times 82 \mathrm{~cm})$ equipped with two rodent levers (Lehigh Valley Electronics, 121-05). One lever was attached approximately $2 \mathrm{in}$. above the wire-mesh floor, in the left rear corner. Depression of this lever initiated a $500-\mathrm{msec}$ train of rectangular cathodal pulses, $.1 \mathrm{msec}$ in duration. The current intensity was manipulated until a high rate of responding was attained.

Stimulation escape. After successful training in the SS paradigm, the animals were trained to escape a 10-sec train of stimulation. Depression of a lever in the diagonally opposite corner from the SS lever terminated the stimulation. When the animal moved to a neutral corner, the stimulation was again initiated. This procedure was repeated until the animal was shaped to the lever. Animals showing severe motor artifacts resulting in the inability to adequately press the lever were discarded from the study.

Once the shaping procedure was completed, the ON time of the stimulating train was fixed at $10 \mathrm{sec}$ and the OFF time was fixed at $1 \mathrm{sec}$. The stimulation was automatically initiated at the start of each trial. A lever depression produced a 1-sec break in the stimulation. If the rat failed to respond, the stimulation was automatically terminated after $10 \mathrm{sec}$ and then reinstated $1 \mathrm{sec}$ later. For each animal, the current intensity that produced optimal SS and SE was determined and used for the remainder of the experiment. The intensities ranged from 300 to $700 \mu \mathrm{A}$.

The stimulation frequency (sf) was decreased every $2.5 \mathrm{~min}$ in $.1 \log _{10}$ steps from the sf that supported maximal responding to that at which the subject failed to respond. Rats were run concurrently in the SS and SE paradigms, on the hour and on the half hour, respectively, over a period of $7 \mathrm{~h}$ per day. In the SS paradigm, 10 priming trains were delivered at the beginning of each 2.5-min step.
Thresholds at each hourly interval were determined as the sf at which the animal responded at one-half of its maximum rate at that hour. Training was continued until thresholds were stable over a 7 -h period on 2 consecutive testing days. The criterion for stabilization was a difference of not more than $.1 \log _{10}$ units between any two hourly tests.

\section{Procedure}

After stabilization of SS and SE, Ringer's solution $(1 \mathrm{ml} / \mathrm{kg})$ was administered to each animal and hourly thresholds were determined. The average threshold for the two Ringer's days was determined for each behavior, and a 6-week series of tests was begun. Although each subject was tested every 5 days, morphine $\mathrm{HCl}$ was administered daily. Injections were always administered at the same hour either in the experimental room (on test days) or in the animal colony.

The testing procedure consisted of 7 identical 1-h cycles; a $1 / 2-h$ test of SS was followed by a $1 / 2-h$ test of SE. A $1-h$ break separated the first and second cycles. Cycles 2-7 were contiguous. A 10-min "warm-up" period during which no data were collected preceded each daily session. The current intensity was held constant across behavior and within animals. Sfs were presented in a descending series of $.1-\log$ steps, with response rates recorded every $2.5 \mathrm{~min}$. If the rat did not respond at a criterion level during the first $\mathbf{2 . 5} \mathrm{min}$, the sf was increased until vigorous behavior was reinstated. The descending series began thereafter.

The effect of the drug on each subject's performance was assessed with the statistic $\log \left(f_{\text {pre }} / f_{\text {post }}\right)$, where $f_{\text {pre }}$ is the average frequency threshold on Ringer's days and $f_{\text {post }}$ is the threshold following drug treatment. This statistic represents the proportional change from the predrug baseline in the frequency required to maintain a half-maximal rate of responding. An increase in this value denotes a facilitatory effect.

\section{Drug Solution}

Morphine hydrochloride was dissolved in injectable Ringer's solution. The initial concentration was $20 \mathrm{mg} / \mathrm{ml}$. At the higher doses (above $80 \mathrm{mg} / \mathrm{kg}$ ), the concentration was increased to $40 \mathrm{mg} / \mathrm{ml}$. After 5 days of injections at a dose of $20 \mathrm{mg} / \mathrm{kg}$ (ip), the dose was incremented in .05-log steps until a dose of $80 \mathrm{mg} / \mathrm{kg}$ was achieved. After 5 days of injections at this dose, the dose was again increased in $.05-\log$ steps until a dose of $320 \mathrm{mg} / \mathrm{kg}$ was achieved. The animals were maintained at this dose for an additional 9 days.

\section{Histology}

At the completion of the experiment, animals were perfused through the heart with $.9 \%$ saline followed by $10 \%$ Formalin. After a minimum of 5 days, the brains were sectioned at $\mathbf{4 0 \mu \mathrm { m }}$ and stained with thionin for histological verification of electrode placements.

\section{Results}

Figure 1 shows reconstructions of electrode placements for five of the six subjects. No histology was available for Animal 34. All electrode tips were located in or on the border of the LH (Pellegrino \& Cushman, 1967).

Figure 2 shows the mean changes in SS and SE as a function of days of treatment and hours since drug administration. The effects of morphine on SS were generally facilitatory with a depression occurring occasionally in the first 1-2 $\mathrm{h}$ following drug administration. An analysis of variance (Days by Hours) revealed a highly significant effect of hour of $\operatorname{SS}[F(6,30)$ $=5.29, \mathrm{p}<.001]$ as well as a significant interaction 


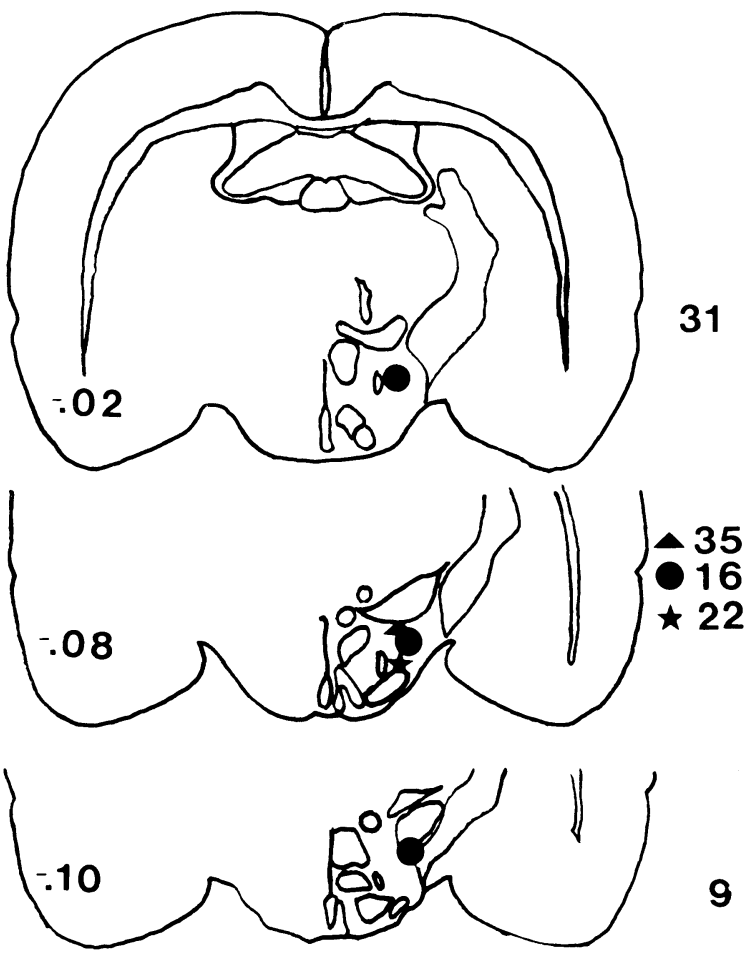

Figure 1. Reconstruction of electrode placements for five of six subjects. No histology was available for Rat 34 . Left-hand numbers refer to the atlas of Pellegrino and Cushman (1967). of Days by Hours $[\mathrm{F}(36,180)=2.89, \mathrm{p}<.001]$.

The interaction effect can be attributed to the variability in the initial depressive effect as a function of days of injections. Tukey post hoc comparisons confirmed the significant facilitatory effects observed following 32,200 , and $320 \mathrm{mg} / \mathrm{kg}(\mathrm{p}<.01)$.

The drug produced a monophasic depression of SE on all test days. This effect usually returned to predrug baseline levels by $3-4 \mathrm{~h}$ postinjection. An analysis of variance (Days by Hours) revealed a significant effect of hours for $\operatorname{SE}[F(6,30)=15.29$, $p<.001]$.

Figure 3 shows changes in behaviors for each subject as a function of hours since drug administration. These data are collapsed across dose in an attempt to illustrate the across-animal variability for SS. In contrast, SE is consistently depressed.

Tolerance effects are shown in Figure 4 for the three doses employed to assess these effects: 20, 80 , and $320 \mathrm{mg} / \mathrm{kg}$. The depression seen in SS at Hour 1 on the first test day at 20 and $320 \mathrm{mg} / \mathrm{kg}$ is seen to tolerate 5 days later on the second test at these doses. The depression of SE shows no such tolerance.

\section{Discussion}

\section{Stimulation Escape}

A significant attenuation of SE was found at all doses tested (Figure 2). This was in accordance with

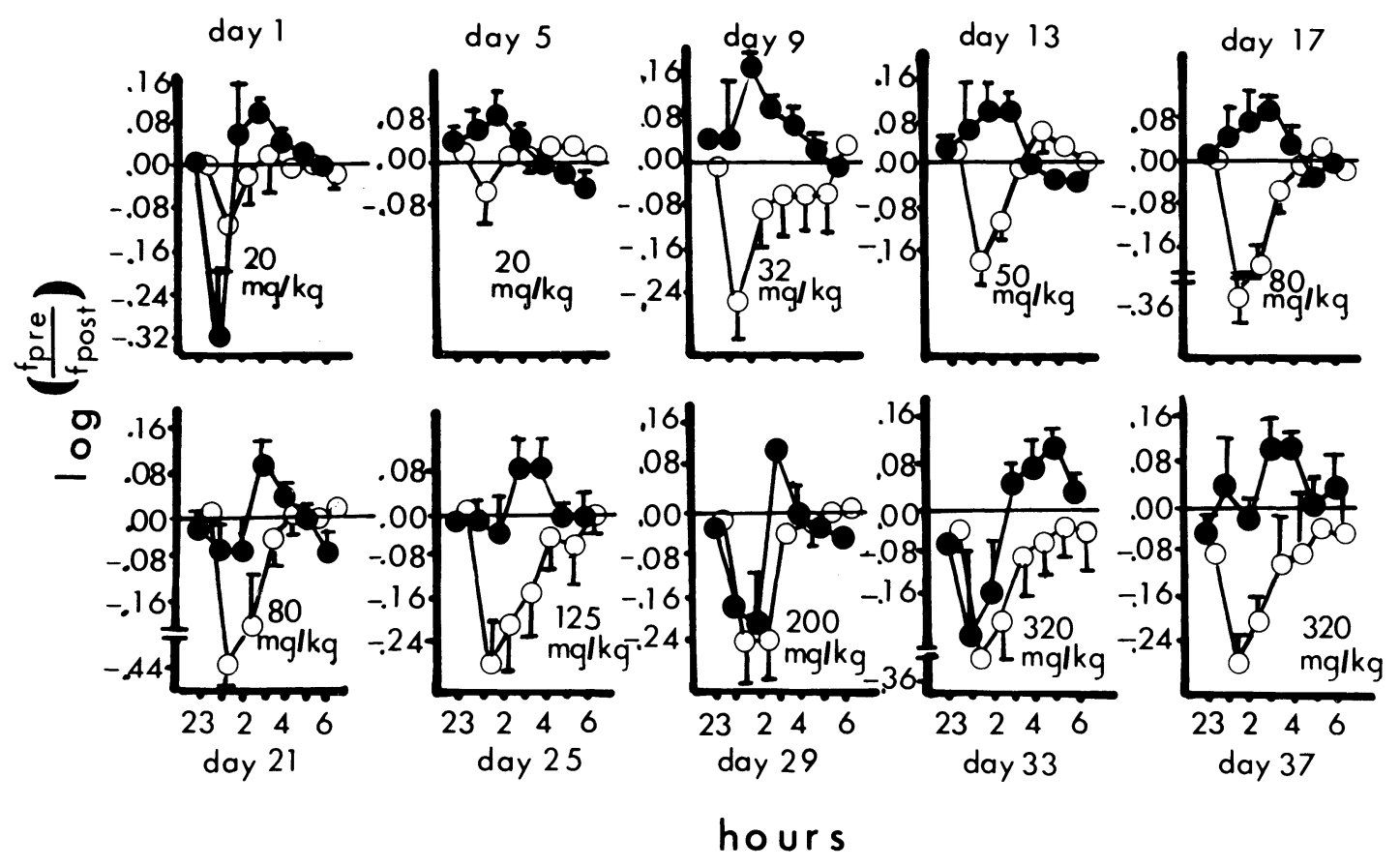

Figure 2. Mean changes in threshold for SS and SE as a function of day of treatment and hours since drug administration. Open circles refer to SE. Closed circles refer to SS. The statistic $\log \left(f_{\text {pre }} / f_{\text {post }}\right)$ reflects the proporional changes in the number of pulses required to maintain behavior following drug treatment as compared with predrug baselines. An increase in this number denotes a facilitatory effect. 


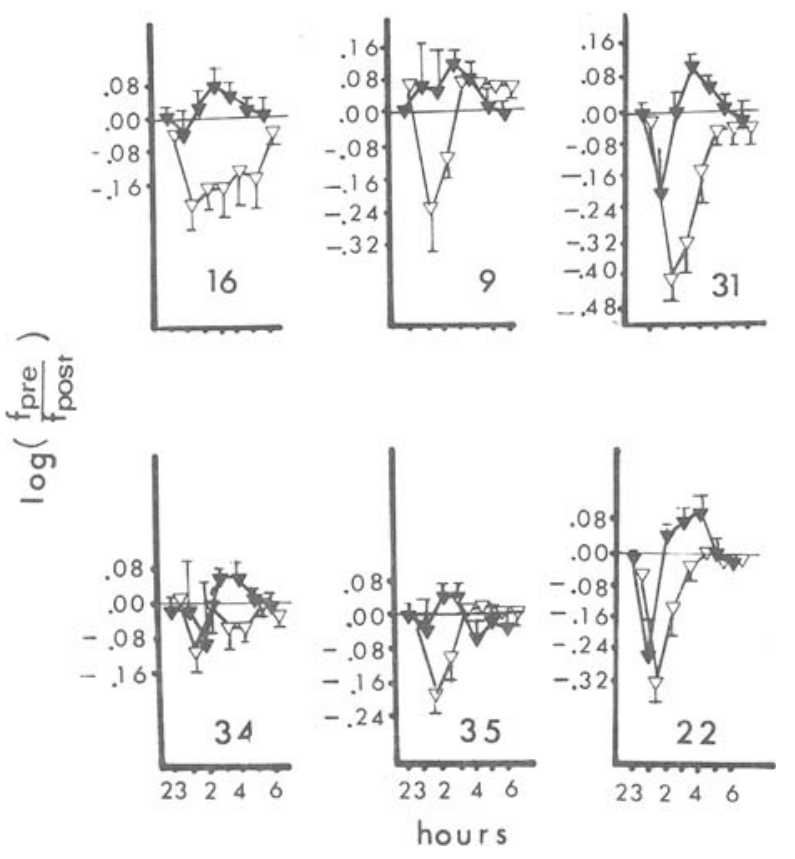

Figure 3. Individual threshold changes for the six subjects. The data are collapsed across days of treatment to illustrate across animal variability. Open triangles refer to SE. Closed triangles refer to SS. Refer to Figure 2 for a description of axes.

the findings of Baltzer et al. (1977) and Marcus and Kornetsky (1974). The lack of tolerance to this effect at a time when depressive effects of SS had tolerated (Figure 4) suggests that this effect of morphine is not due to motoric disruption but, rather, to a desensitization of the neural substrate mediating the aversive consequences of brain stimulation.

The lack of tolerance to the attenuation of SE is contrary to Pert's (1975) findings. These differences may be attributed to differences in the stimulation site. Pert used the superior colliculus as the negative stimulation site. Kestenbaum, Deutsch, and Coons (1974) have shown that there is behavioral adaptation to the aversion produced by stimulation of some midbrain sites. In contrast, Shizgal and Matthews (1977) have demonstrated that the aversive effects of LH stimulation accrue over time. The effects at the superior colliculus site may be related to morphine analgesia, which also shows tolerance with repeated injections. The lack of tolerance to the morphineproduced attenuation of LH SE suggests that processes subserving analgesia were not involved in the drug's effect at this site.

\section{Self-Stimulation}

The effect of morphine on SS was less consistent than the drug's effects on SE. The reasons for such individual differences are unclear. Figure 3 illustrates these inconsistencies. Two subjects showed the classic biphasic effect, while the others showed varying facilitatory effects on SS with large depressions of SE. A similar degree of variability has been demonstrated in morphine self-administration as well as in the conditioned taste aversion paradigm (Amit, Note 1) which are also claimed to measure affective states associated with drug administration. These differences may be due to individual subjects' different susceptibilities to the drug's reinforcing effects. It is also possible that differences in placement may account for the individual differences observed. These possibilities are currently under investigation in our laboratory.

In summary, it seems that the neural systems underlying the appetitive and aversive properties of $\mathrm{LH}$ stimulation are both sensitive to morphine. Due to differences in the time course of the two effects, as well as to differences in across-animal variability,
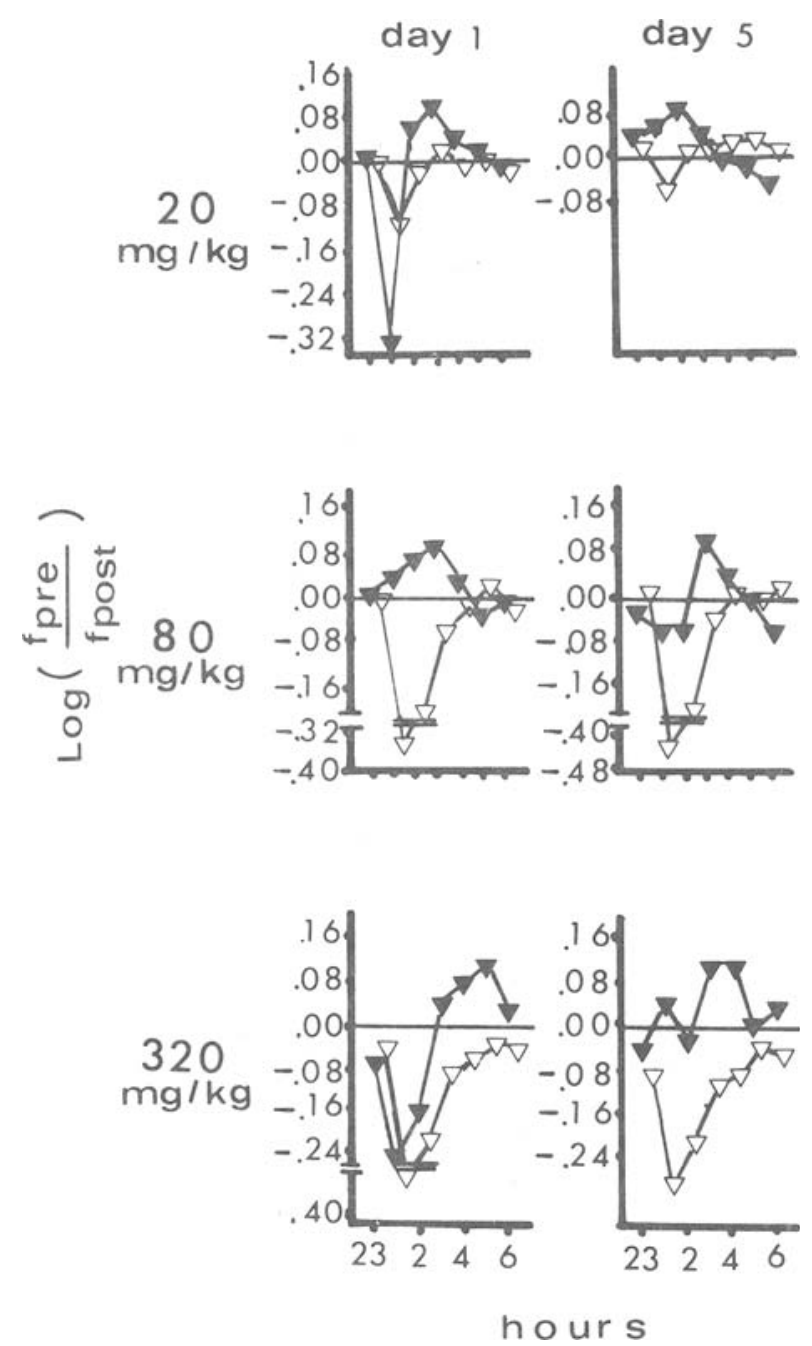

Figure 4. Tolerance effects of SS (closed triangles) and SE (open triangles) for the three doses used to assess these effects. 
it is unlikely that the attenuation of SE is responsible for the facilitation of SS, or vice versa. Rather, it seems that the underlying systems are both affected by the drug.

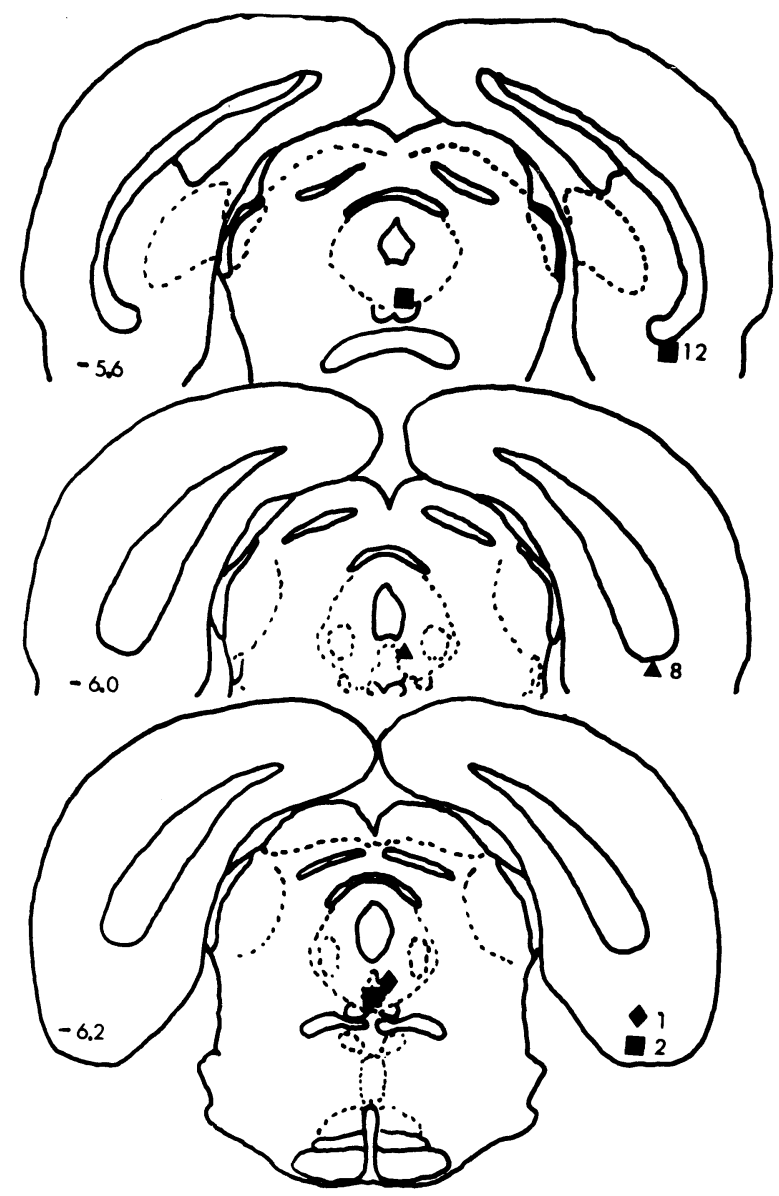

Figure 5. Reconstruction of electrode placements for the four subjects. Left-hand numbers refer to the atlas of Pellegrino and Cushman (1967).

\section{EXPERIMENT 2}

This experiment was designed to investigate the effects of morphine on the rewarding and aversive properties of DR stimulation and to compare these findings with those observed in the $\mathrm{LH}$.

\section{Method}

\section{Subjects}

The subjects were male Sprague-Dawley rats, weighing approximately $300 \mathrm{~g}$ at the time of surgery. Electrodes, surgery, and apparatus were the same as those described in Experiment 1, except that the electrodes were aimed at the DR (AP, $-6.0 \mathrm{~mm}$; lat, $.0 \mathrm{~mm} ; 6.0 \mathrm{~mm}$ below the dura; incisor bar at $+5.0 \mathrm{~mm}$ ).

\section{Procedure}

The procedure was exactly the same as that described in Experiment 1 except that the DR was tested instead of the LH. Since no significant effect of dose was observed in Experiment 1, this experiment was confined to the lowest dose used, $20 \mathrm{mg} / \mathrm{kg}$. The animals were injected with this dose of the drug for 5 days and tested on Days 1, 3, and 5 of that sequence.

\section{Results}

In four subjects, SS and SE were readily achieved. However, six other subjects, after attaining vigorous SS, had to be discarded from the study due to severe motor artifacts or freezing behavior with prolonged stimulation.

Figure 5 is a reconstruction of the electrode placements for the four subjects. According to the atlas of Pellegrino and Cushman (1967), two electrode tips (Rats 2 and 12) were located in the region of the DR. The other two (Rats 1 and 8) were located dorsolateral to the $\mathrm{DR}$, in the region of the periaqueductal gray.

The mean changes in SS and SE threshold as a function of days of treatment and hours since drug administration are presented in Figure 6. A monophasic depression of SE is observed at Hours 1 and 2
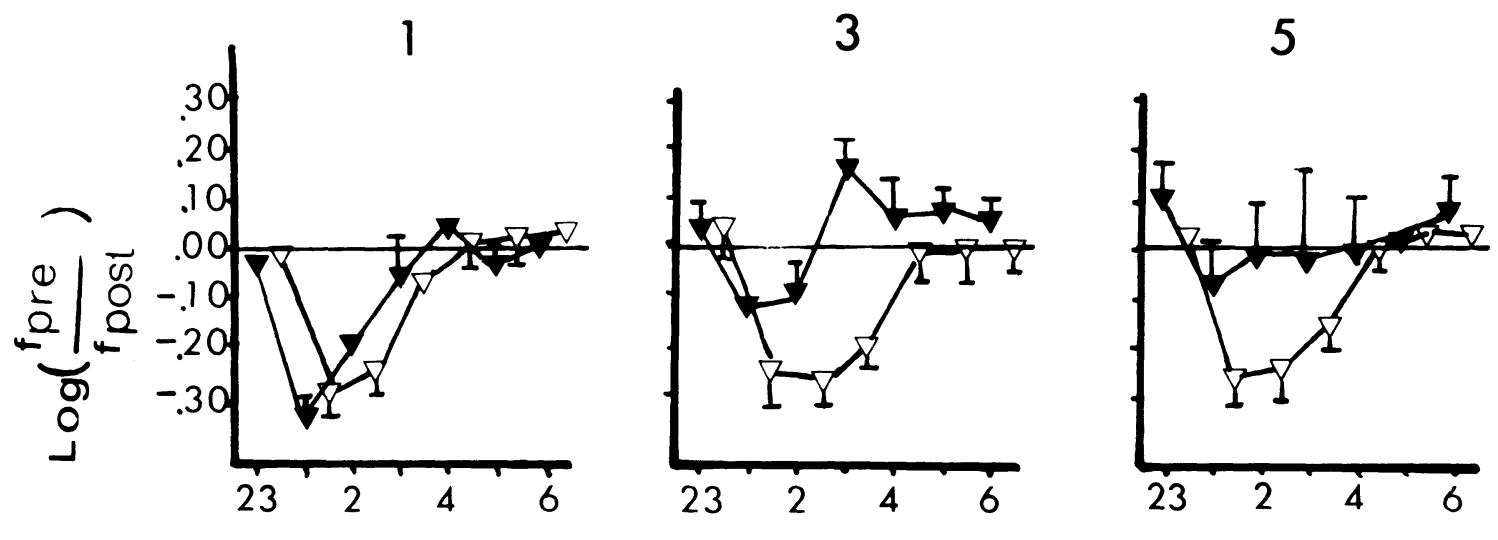

\section{hours}

Figure 6. Mean changes in threshold for SS (closed triangles) and SE (open triangles) as a function of days of treatment and hours since drug administration. Refer to Figure 2 for a description of axes. 


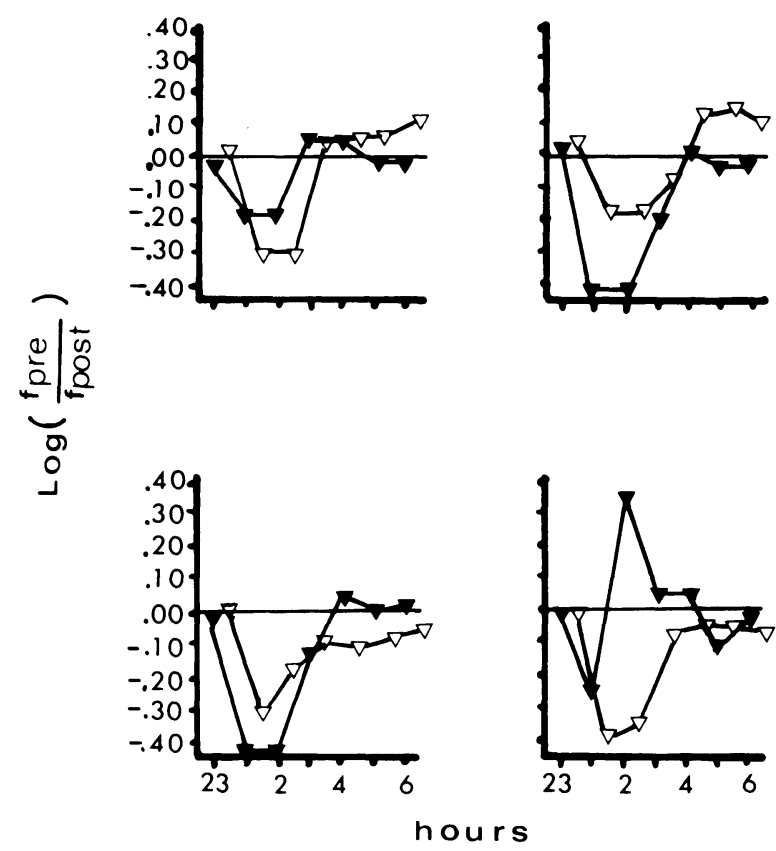

Figure 7. Individual threshold changes for the four subjects on Day 1 of drug treatment. Open triangles refer to SE. Closed triangles refer to SS. See Figure 2 for a description of the axes.

postinjection on all test days. An analysis of variance (Days by Hours) for repeated measures confirmed this significant hours effect $[F(6,18)=17.91, p<.001]$. The SS data are more variable. There is a large facilitatory effect on Day 3. Due to high variability, this effect is not apparent on Day 5. This variability can also account for the lack of a significant hours or interaction effect in an analysis of variance. Two measures of SS were discarded from the analysis for one subject due to a high level of nonreinforced responding (gnawing) on Day 5.

To illustrate the individual animal data, Figure 7 shows changes in behavior for each of the subjects on the first test day. One of the four subjects showed a facilitatory effect on SS at Hour 2.

\section{Discussion}

Our difficulty in training subjects to escape DR stimulation does not appear to have been shared by Steiner et al. (1973), who used bursts of stimulation instead of uninterrupted trains. It is possible that the bursts interfered less with the leverpressing response.

The strong drug-produced depression of SE was different both in time course and in across-subject variability to the facilitation observed in SS, again suggesting a sensitivity to the drug of both neural substrates. The average curves (Figure 6) indicate a high degree of variability, with only one subject showing a large facilitatory effect on SS (Figure 7), in contrast with the consistency of SE.

\section{GENERAL DISCUSSION}

The SS data, at both placements, were highly variable. Some subjects showed facilitatory effects of varying magnitude, while others showed only depressions. Due to this high variability, it is difficult to compare effects at these two sites. A within-animal comparison study, examining individual-subject SS thresholds at these two placements may help to determine whether the systems are equally sensitive to the drug's facilitatory effects, as well as the source of the variability observed.

In contrast, a significant attenuation of $\mathrm{SE}$, of similar duration, was found in all animals, on all test days, at both placements. This finding lends itself to several interpretations. It is possible that the same directly stimulated neurons underlie the aversive consequences of stimulation at both neural sites, and that morphine acts upon these cells. In support of this hypothesis is the recent finding that DR lesions can modulate escape from medial hypothalamic stimulation (Schmitt, Paunovic, \& Karli, 1979). Alternatively, the stimulation at these two placements may excite different neurons, with morphine acting on both with a similar time course. Another possibility is that different converging fiber bundles are activated by the stimulation. Morphine may then act beyond the proposed point of convergence, thereby producing a similar effect on SE from LH and DR stimulation.

Using the pulse-pair technique (Shizgal, Bielajew, Corbett, Skelton, \& Yeomans, 1980), the manner in which the neurons mediating the aversive consequences of stimulation link these two sites is currently being investigated in our laboratory. Such data may choose between the possibilities outlined above.

\section{REFERENCE NOTE}

1. Amit, Z. Personal communication, 1978.

\section{REFERENCES}

Baltzer, J. H., Levitt, R. A., \& Furby, J. E. Etorphine and shuttle-box self-stimulation in the rat. Pharmacology, Biochemistry and Behavior, 1977, 7, 413-416.

Bielajew, C., \& Shizgal, P. Dissociation of the substrates for medial forebrain bundle self-stimulation and stimulation-escape using a two-electrode stimulation technique. Physiology \& Behavior, in press.

Esposito, R. U., \& Konnetsky, C. Opioids and rewarding brain stimulation. Neuroscience and Biobehavioral Review, 1978, 2, 115-122.

Jackler, F., Steiner, S. S., Bodnar, R. J., Arkermann, R. F., Nelson, W. T., \& Ellman, S. J. Morphine and intracranial self-stimulation in the hypothalamus and dorsal brainstem: Differential effects of dose, time, and site. International Journal of Neuroscience, 1979, 9, 21-35.

Kestenbaum, R. S., Deutsch, J. A., \& Coons, E. E. Inference of refractory period, temporal summation, and adaptation from behavior in chronic implants. Journal of Comparative and Physiological Psychology, 1974, 83, 412-428.

Liebman, J., \& Segal, D. S. Differential effects of morphine 
and d-amphetamine on self-stimulation from closely adjacent regions in rat midbrain. Brain Research, 1977, 136, 103-117.

Lorens, S. A. Comparison of the effects of morphine on hypothalamic and medial frontal cortex self-stimulation in the rat. Psychopharmacology, 1976, 48, 217-224.

Marcus, R., \& Kornetsky, C. Negative and positive intracranial reinforcement thresholds: Effects of morphine. Psychopharmacology, 1974, 38, 1-13.

Mayer, D. J., \& Price, D. D. Central nervous system mechanisms of analgesia. Pain, 1976, 2, 379-404.

Pellegrino, L. J., \& Cushman, A. J. A stereotaxic atlas of rat brain. New York: Appleton-Century-Crofts, 1967.

Pert, A. Effects of opiates on rewarding and aversive brain stimulation in the rat. Proceedings of the 37th Annual Scientific Meeting Committee on Drug Dependence, National Academy of Science, 1975, pp. 963-973.

Poschel, B. P. H., \& Ninteman, F. W. Intracranial reward and the forebrain serotonergic mechanisms: Studies employing parachlorophenylalanine and parachloroamphetamine. Physiology \& Behavior, 1971, 7, 39-46.

Robertson, A., Kucharczyk, J., \& Mogenson, G. J. Selfstimulation of the subfornical organ and lateral hypothalamus: Differential effects of atropine and methysergide. Pharmacology, Biochemistry and Behavior, 1977, 7, 173-176.

Schmitt, P., Pannovie, V. R., \& Karli, P. Effects of mesencephalic central gray and raphé nuclei lesions on hypothalamically induced escape. Physiology \& Behavior, 1979, 23, 85-95.

Shizgal, P., Bielajew, C., Corbett, D., Skelton, R., \&
YEOMANS, J. Behavioral methods for inferring anatomical linkage between rewarding brain stimulation sites. Journal of Comparative and Physiological Psychology, 1980, 94, 227-237.

Shizgal, P., \& Matthews, G. Electrical stimulation of the rat diencephalon: Differential effects of interrupted stimulation on on- and off-responding. Brain Research, 1977, 129, 319-333.

Simon, H., Le Moal, M., \& Cardo, B. Intracranial selfstimulation from the dorsal raphé nucleus of the rat: Effect of the injection of para-chlorophenylalanine and of alphamethylparatyrosine. Behavioral Biology, 1976, 16, 353-364.

Steiner, S., Bodnar, R., Ackermann, R., \& Ellman, S. Escape from rewarding brain stimulation of dorsal brainstem and hypothalamus. Physiology \& Behavior, 1973, 11, 589-591.

Tatum, A. L., Seevers, M. H., \& Collins, K. H. Morphine addiction and its physiological interpretation based on experimental evidence. Journal of Pharmacology and Experimental Therapeutics, 1929, 36, 447-475.

Wauquier, A., \& Niemegeers, C. J. E. Intracranial selfstimulation in rats as a function of various stimulus parameters: Influence of fentanyl, piritramide, and morphine. Psychopharmacologia, 1976, 46, 179-183.

Yeomans, J. S. Quantitative measurement of neural poststimulation excitability with behavioral methods. Physiology \& Behavior, 1975, 15, 593-602.

(Received for publication October 23, 1979; revision accepted April 28, 1980.) 